\title{
Phenotypic Marker Expression during Fetal and Neonatal Differentiation of Rat Tracheal Epithelial Cells
}

\author{
Scott H. Randell, Takeshi Shimizu, William Bakewell, Frans C. S. Ramaekers, \\ and Paul Nettesheim
}

Laboratory of Pulmonary Pathobiology, National Institute of Environmental Health Sciences, Research Triangle Park, North Carolina; Department of Otorhinolaryngology, Mie University School of Medicine, Mie, Japan; and Department of Molecular Cell Biology and Genetics, University of Limburg, Maastricht, The Netherlands

\begin{abstract}
The expression of phenotypic markers was examined during fetal and neonatal differentiation of rat tracheal epithelial (RTE) cells. The rat counterpart of human keratin 18 was predominantly found in columnar cells in the adult trachea. It was detected in the primordial tracheal epithelium first seen on gestational day (GD) 12 (term $=21.5$ days). Staining intensity gradually increased, and by GD 17 it was principally localized to the apical portion of the epithelium. The rat counterpart of human keratin 19 was barely detectable in the trachea on GD 13 but became abundant in almost all RTE cells on and after GD 19. Morphologically and immunocytochemically identifiable secretory and ciliated cells appeared on GD 18. Ciliated cell number slowly rose while secretory cells increased dramatically on GD 19 through postnatal day 1. The secretory granule antigens detected by monoclonal antibodies RTE 9 and 11 were rare in the adult trachea but were highly expressed in virtually all of the perinatal secretory cells. In contrast, the epitope detected by monoclonal antibody RTE 12 , which was present in all adult tracheal surface secretory cells, did not appear until postnatal day 1 and slowly increased. These results demonstrate marked shifts in the biochemical composition of secretory cells during development and postnatal maturation. For the abovementioned molecules, a similar expression pattern was observed during epithelial regeneration in tracheal grafts (Am. J. Respir. Cell Mol. Biol. 1992; 7:30-41). Pseudostratification of the epithelium and basal cells was first observed on GD 20. Keratin 14, which is confined to basal cells in the normal adult trachea, was not present in the nascent basal cells but appeared after postnatal day 1 . In contrast to the present results, during epithelial regeneration in tracheal grafts keratin 14 appeared before markers of highly differentiated secretory or ciliated cells. Thus, the biochemical sequence of cellular differentiation during regeneration did not precisely recapitulate development.
\end{abstract}

Exact pathways of cellular biochemical differentiation during tracheal epithelial development have not been elucidated, but it is thought that basal, secretory, and ciliated cells arise from an undifferentiated precursor $(1,2$ [and references within]). Secretory cells are highly proliferative during development and may give rise to ciliated cells $(1,2)$. Typical basal cells appear late in gestation and thus are not the progenitors of secretory or ciliated cells (1-3).

Molecules such as keratins and specific sugar residues on glycoconjugates that are expressed in distinct morphologic

(Received in original form August 17, 1992 and in revised form November 25, 1992)

Address correspondence to: Dr. Scott H. Randell, NIEHS, P.O. Box 12233, LPP, MD D2-01, Research Triangle Park, NC 27709.

Abbreviations: alcian blue-periodic acid-Schiff-hematoxylin, AB-PAS-H; B5 solution plus glutaraldehyde, B5-G; postnatal day, D; glutaraldehyde, GA; gestational day, GD; Griffonia simplicifolia I-isolectin $\mathrm{B}_{4}$, GS I-B $\mathbf{4}_{\mathbf{4}}$; monoclonal antibody, mAb; poorly differentiated, PD; paraformaldehyde, PFA; rat tracheal epithelial, RTE.

Am. J. Respir. Cell Mol. Biol. Vol. 8. pp. 546-555, 1993 cell types in the normal adult rat trachea can be detected with monoclonal antibodies (mAbs) and lectins (4-7). The expression of several of these markers was recently examined during epithelial regeneration in tracheal grafts (7). A cell provisionally called the "poorly differentiated" cell (PD cell) was a pivotal intermediate. Interestingly, PD cells expressed keratin 14 and Griffonia simplicifolia I-isolectin B $_{4}$ (GS $\mathrm{I}-\mathrm{B}_{4}$ ) lectin binding sites, which are features of basal cells in adult tracheas, but they did not express markers characteristic of highly differentiated secretory or ciliated cells. Differentiation of specific cellular phenotypes from PD cells involved the gradual loss of keratin 14 and GS I-B 4 lectin binding sites and the acquisition of molecules characteristically expressed in secretory and ciliated cells.

The purpose of the studies described here was to better understand the evolving cellular biochemical phenotype of the developing tracheal epithelium and to compare the sequence of phenotypic marker expression between development and regeneration. mAb RTE 1 , which was produced in our laboratory, detects an antigen found in almost all adult rat tracheal epithelial (RTE) cells (5). We demonstrate that 
RTE 1 detects the rat counterpart of human keratin 19. The ontogeny of this protein was examined, as was the developmental expression of the rat equivalents of human keratins 18 and 14 , which are predominantly localized to columnar and basal RTE cells, respectively (6). Temporal expression patterns of phenotypic markers for subtypes of adult airway secretory cells as well as ciliated cells were also evaluated.

\section{Materials and Methods}

\section{Animals}

Timed-pregnant $\mathrm{F} 344$ rats from the NIEHS breeding colony were used under an Institutional Animal Care and Use Committee-approved protocol. Sperm-positive vaginal washings after an overnight period of cohabitation with a male was considered evidence of pregnancy; the midpoint of cohabitation was counted as the beginning of the 21.5-day average gestational period of these animals.

\section{Preparation of Fetal and Neonatal Rat Tissues}

Two pregnant rats were killed on gestational days (GD) 12 to 20 and four to six pups were killed on postnatal days (D) $1,7,14,21$, and 28 using $\mathrm{CO}_{2}$ asphyxiation or sodium pentobarbital overdose. Fetuses were removed, and fetal tissues were obtained for histologic preparation. Up to GD 18, the entire fetus was immersed in fixative (fixatives specified below); thereafter, tracheas were removed for fixation. Two to four tracheas per time point for each fixative were obtained. Samples representing the upper and lower trachea were prepared for histochemical and immunohistochemical staining procedures as recently described $(4,5,7)$. Briefly, five different fixation-embedding combinations were used: $(I)$ fixation with $2 \%$ paraformaldehyde (PFA), $2 \%$ glutaraldehyde (GA), and methacrylate embedding (JB-4 embedding kit; Polysciences, Warrington, $\mathrm{PA}$ ) for alcian blue ( $\mathrm{pH} \mathrm{2.5)-periodic}$ acid-Schiff-hematoxylin (AB-PAS-H) staining; (2) acetone fixation and paraffin embedding for staining with mAbs RTE $1,9,11,2,7$, and 13, LLO02, and CK18-2 (all antibodies are described below); (3) 4\% PFA fixation and paraffin embedding for staining with mAb RTE 3; (4) 4\% PFA fixation and frozen sectioning for staining with mAb RTE 12; (5) fixation with B5 solution (Polysciences) to which $0.1 \%$ GA was added (B5-G) and paraffin embedding for lectin histochemistry. Two-, five-, and eight-micron-thick cross sections were obtained using JB-4-embedded, paraffin-embedded, or frozen tissues, respectively.

\section{Phenotypic Markers for RTE Cells}

Eight different lectins or mAbs whose reactivity with adult RTE cells has been characterized previously (4-6) were utilized. The staining methods were similar to those previously described $(4,5,7)$, with the exception that trypsin-EDTA pretreatment $(0.025 \%$ and $0.27 \mathrm{mM}$, respectively; $10 \mathrm{~min}$ at $25^{\circ} \mathrm{C}$ ) was used before $\mathrm{mAb}$ CK18-2 and RTE 1 staining and $5 \%$ normal goat serum and $5 \%$ gelatin were used in the blocking step. The reagents used and their staining properties in the normal adult trachea are discussed below and are summarized in Table 1. With regard to the anti-keratin antibodies, it should be noted that there is no keratin catalog for rats as for humans (8); we do not know how many rat keratins exist. In strict terms, the rat proteins detected by anti-human keratins are "human keratin counterparts in rats." For simplicity and brevity, throughout this manuscript, we will refer to them by their respective human keratin catalog numbers (8). mAb CK18-2 detects human keratin 18 (9) and strongly stains columnar-type epithelial cells in the adult rat trachea. mAb RTE 1 stains almost all RTE cells and epithelial cells in several other organs but does not stain any mesenchymal tissues (5); as shown below, it detects rat keratin 19. $\mathrm{mAb}$ RTE 3 intensely stains the cilia and apical plasma membrane of ciliated cells (5). mAbs RTE 9 and 11 react with the granules of all mucous-type adult tracheal secretory cells and with a small subpopulation of serous-type cells (5). mAb RTE 12 stains the granules of almost all tracheal surface secretory cells, either mucous or serous types, and intestinal goblet cells, but does not stain bronchial epithelial cells (5). GS I-B ${ }_{4}$ lectin is relatively specific for basal cells in the adult rat trachea. As previously described (4), all tracheal basal cells were stained and $84 \%$ of GS I- ${ }_{4}$-positive tracheal cells were basal cells; however, $7 \%$ were unidentified cells, $5 \%$ were secretory cells, and $4 \%$ were ciliated, brush, or inflammatory cells. mAb LL002 reacts with human keratin $14(10)$ and is specific for basal cells in the adult rat trachea (6). mAbs RTE 2, 7, and 13 react with most nonciliated cells, both basal and columnar-type cells, in the upper trachea; they recognize an abundant $69 \mathrm{kD}$ protein and several lower molecular weight bands on immunoblots of RTE cells, but the antigen(s) is either masked or absent in the lower trachea and bronchi (5).

\section{Biochemical Characterization of the Antigen for mAb RTE 1}

Previous immunocytochemical and immunoblot results with mAb RTE 1 suggested that it detected one specific keratin.

TABLE 1

Phenotypic markers for RTE cells

\begin{tabular}{lll}
\hline Probe & Epitope & Staining Pattern in Normal Adult Rat Trachea \\
\hline 1. mAb CK 18-2 & Keratin 18 & Predominantly columnar cells \\
2. mAb RTE 1 & Keratin 19 & Most cells \\
3. mAb RTE 3 & Unknown & Ciliated cell plasma membrane \\
4. mAbs RTE 9 and 11 & Unknown & Mucous cells, small subpopulation of serous cells \\
5. mAb RTE 12 & Unknown & All tracheal surface secretory cells \\
6. GS I-B 4 lectin & $\alpha 1,3$ gal* & Predominantly basal cells \\
7. mAb LLOO2 & Keratin 14 & Basal cells \\
8. mAbs RTE 2, 7, and 13 & Unknown & Nonciliated cells in upper trachea \\
\hline
\end{tabular}

$* \alpha 1,3$ gal $=\alpha 1,3$ linked terminal galactose. 

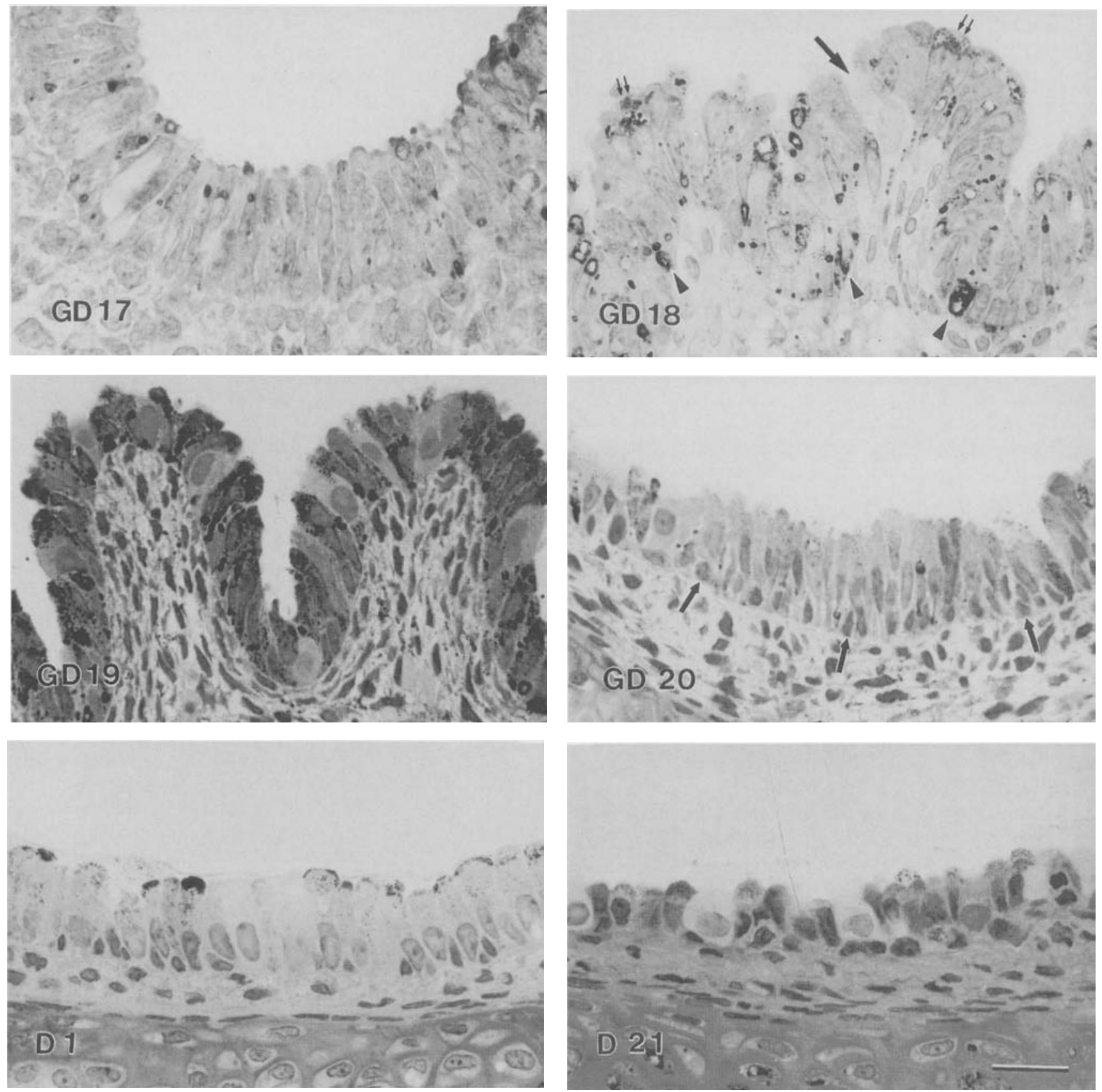

Figure 1. Histochemical staining of developing rat trachea. Two-micron-thick methacrylate sections, AB-PAS stain. Glycogen droplets (arrowheads), preciliated cells (arrow), and secretory cells (double arrows) were visible on GD 18. There was a rapid increase in the number of preciliated and secretory cells by GD 19. Clear evidence of a distinct basal cell layer (arrows) and pseudostratification were visible on GD 20. Bar $=20 \mu \mathrm{m}$. GD 17 to 19 : membranous area; GD 20 to D'21: cartilaginous area.

We used a "hot blot" immunoblot technique to characterize the antigen. Primary RTE cells were grown for 14 days in membrane-interface cultures in media containing retinoic acid as previously described (11). Under these conditions, the cells underwent mucociliary differentiation, and keratin expression closely resembled that of RTE cells in vivo (11). After a 1-h preincubation period in methionine-free medium, the cells were incubated in medium containing $100 \mu \mathrm{Ci} / \mathrm{ml}$ of $\left[{ }^{35} \mathrm{~S}\right]$ methionine for $4 \mathrm{~h}$. Cytoskeletal extracts were pre- pared (12) and samples were subjected to two-dimensional electrophoresis using isoelectric focusing in the first dimension and sodium dodecyl sulfate polyacrylamide gel electrophoresis in the second (13). Proteins were transferred to nitrocellulose, and autoradiographs were prepared. The membrane was then probed with $\mathrm{mAb}$ RTE 1 or $\mathrm{MAb}$ LP2K, against human keratin 19 (14). Antibody binding was detected with a peroxidase-labeled second antibody as described (5). Using this technique, we were able to directly 


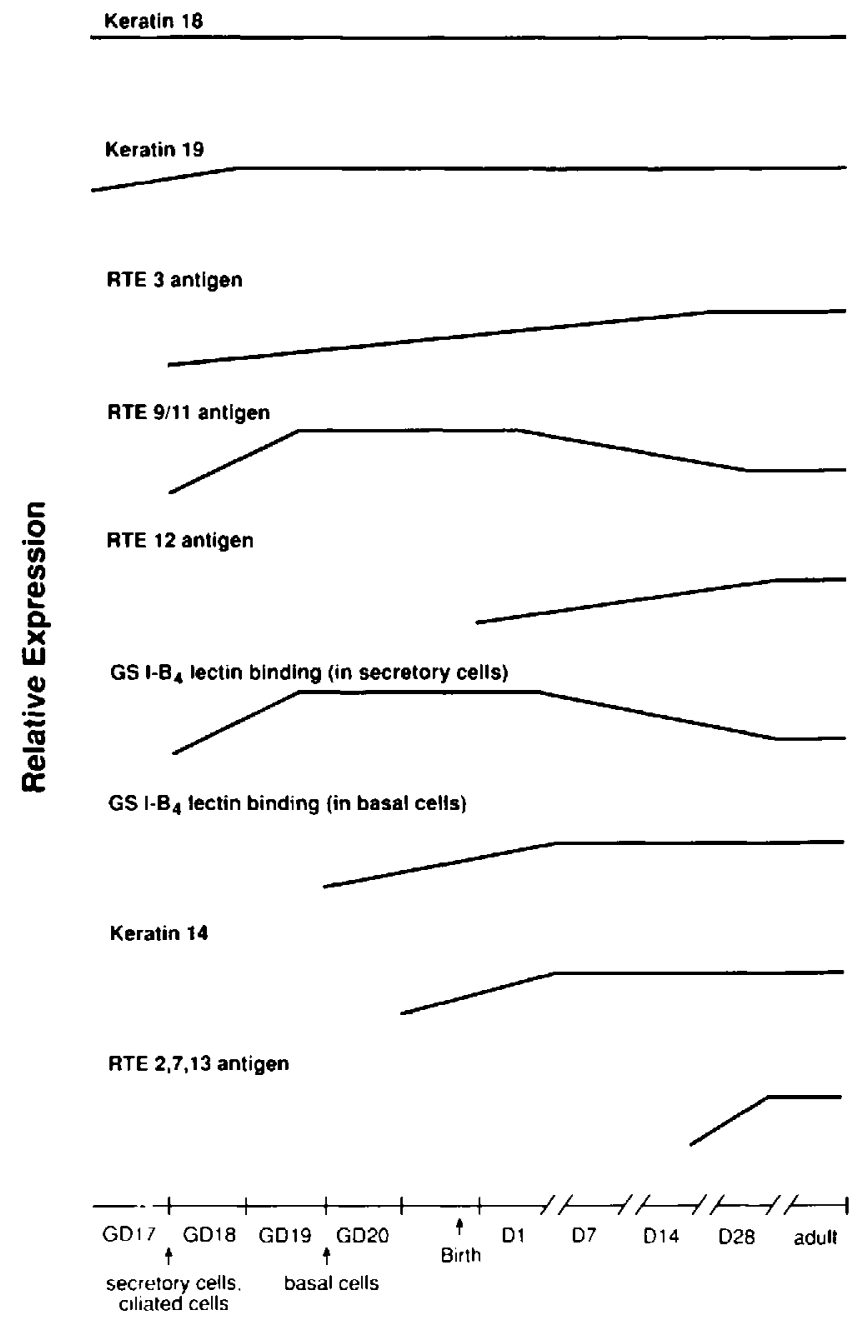

Figure 2. Graphic representation of phenotypic marker expression during rat tracheal development. The term "relative expression" reflects both the percentage of the total tracheal epithelial cells stained and the intensity of staining.

compare the total cytoskeletal protein pattern on the autoradiogram with the specific spot stained by the antibodies on the immunoblot.

\section{Results}

\section{Morphologic Evaluation of the Developing} Trachea with AB-PAS-H Staining

Cytologic development of the trachea was assessed with 2- $\mu \mathrm{m}$-thick AB-PAS-H-stained methacrylate sections to provide a framework for analysis of marker expression. As discussed below, RTE cells remained relatively undifferentiated before GD 18, and morphologic analysis was focused on the perinatal period. Representative photomicrographs are shown in Figure 1.

Undifferentiated cells. On and before GD 17, the tracheal epithelium was of the simple columnar type and consisted primarily of undifferentiated tall and narrow cells with a central nucleus. Undifferentiated cells did not display secretory, ciliated, or basal cell characteristics but contained widely dispersed, PAS-positive cytoplasmic droplets, which were shown to be glycogen by their diastase digestibility.

Ciliated cells. On GD 18, a few large cells with a broad luminal border, lightly stained cytoplasm, and a large, round nucleus were observed overlying the membranous area of the trachea; these cells were PAS-negative and did not have cilia. By GD 19, short, immature cilia were visible at the luminal border of these morphologically distinct cells. On GD 20 to 21 , ciliated cells were still few in number and were observed only over the membranous portion of the trachea. On D 1, ciliated cells were still sparse, but they were now found in the epithelium overlying cartilaginous areas. By D 7, the relative frequency of ciliated cells increased and by D 21 almost the entire luminal surface was covered with cilia.

Secretory cells. On GD 18, PAS-positive, diastase-resistant secretory granules first appeared in the apical cytoplasm of a few cells overlying the membranous area of the trachea. These granules were distinguishable from glycogen accumulations by their visible hematoxylin-stained corc structures, smaller size, and apical clustering. The cytoplasms of the nascent secretory cells were also stained darkly with hematoxylin, contained glycogen, and the cell apex protruded into the airway lumen. On GD 19, the number of PAS-positive, diastase-resistant secretory granules per cell increased. Secretory granules appeared in the apical cytoplasm of almost all nonciliated columnar cells overlying both the membranous and cartilaginous areas, and cellular glycogen content was high. The amount of diastase-resistant, PASpositive materials increased dramatically on GD 20 and on D 1. At this time, the apical cytoplasms of secretory cells as well as their granules were PAS-positive. Cells containing AB-positive granules were first observed on D 1. By D 7, the diffuse PAS-reactivity of the apical cytoplasm of secretory cells disappeared and PAS staining was confined to granules. By D 14 and 21, secretory cell frequency and morphology approached the adult values.

Basal cells. On GD 19, the epithelium was still of the simple columnar type, almost all cells had centrally placed nuclei, and no basal cells were observed. On GD 20, pseudostratification of the epithelium began and morphologically identifiable basal cells were observed. On D 1, the epithelium was pseudostratified and the nuclei of all cells were located in the lower third of the epithelium. Basal cells were clearly recognizable on and after D 1 .

\section{Expression of Phenotypic Markers}

The time-dependent expression of phenotypic markers is discussed below in order of developmental appearance and the results are summarized in Figure 2.

Keratin 18. mAb CK18-2, which reacts with human keratin $18(8)$, predominantly stained columnar cells of the adult rat trachea (6); expression during development is illustrated in Figure 3. All the primordial tracheal epithelial cells first seen on GD 12 were stained. At early times when the expression was low, mainly cytoplasmic rims at the cell apex and base were stained. Staining intensity gradually increased and by GD 17 the protein became principally localized to the apical cytoplasmic portion of the epithelium. Thereafter, staining was detected in all columnar cells, both ciliated and 

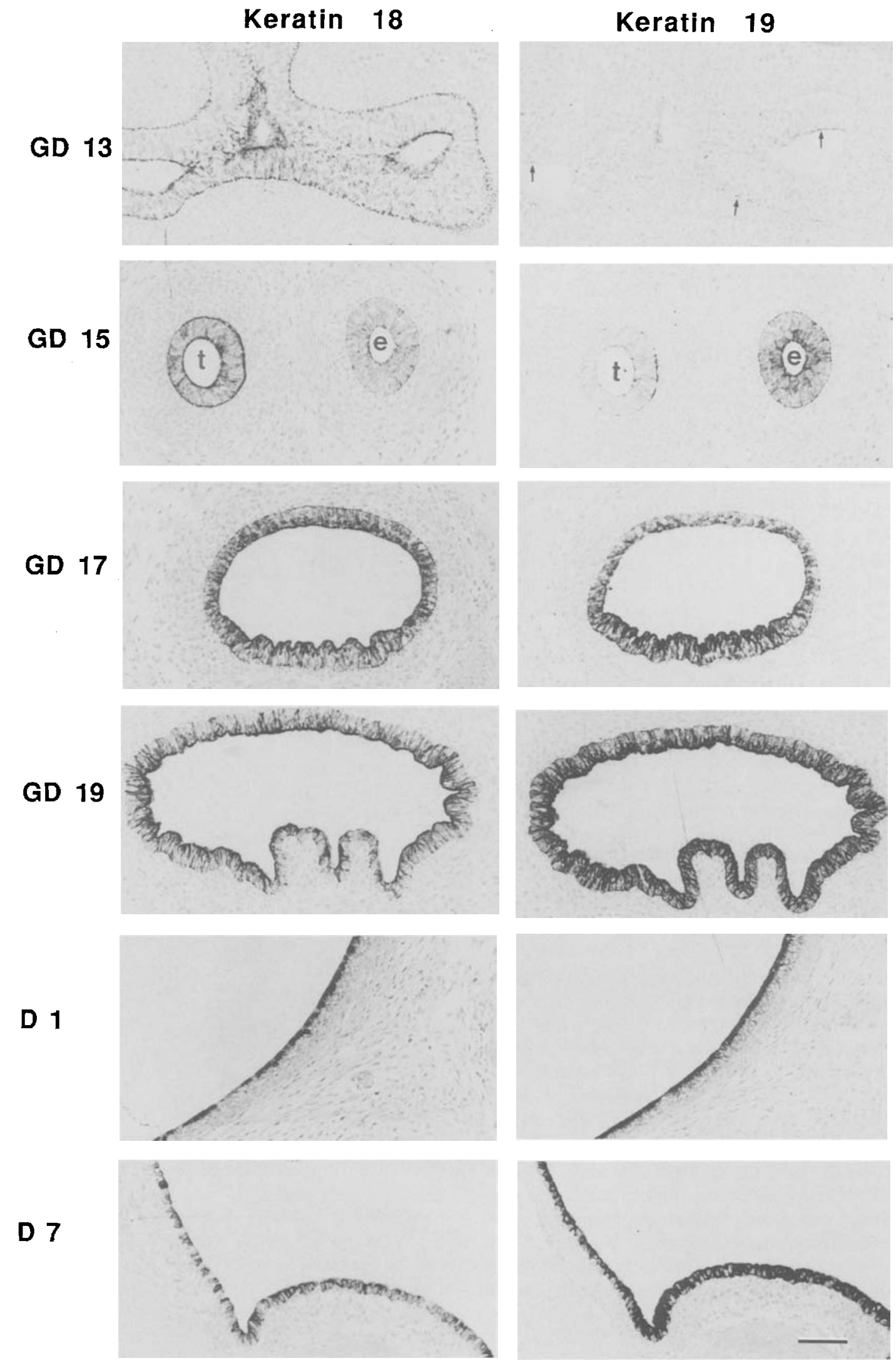

Figure 3. Keratin 18 and 19 protein expression during tracheal development. mAb CK18-2 and mAb RTE 1 immunoperoxidase-stained sections, methyl green counterstain. Both keratins were present in the tracheal and lung primordium on GD 13 , but keratin 19 staining was very faint (arrows). On GD 15, keratin 18 staining was strong in the trachea (t), but keratin 19 staining was relatively weak; the developing esophagus (e) is also shown. Keratin 18 and 19 were both abundant from GD 18 onward. Bar $=50 \mu \mathrm{m}$. 

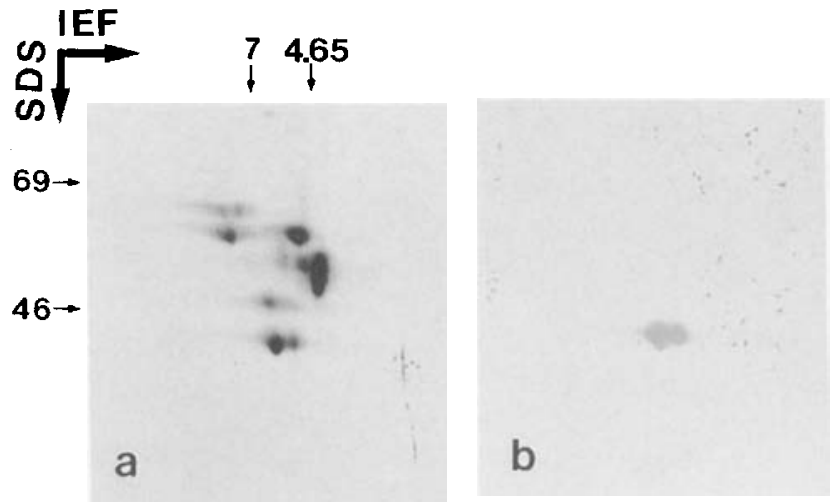

Figure 4. mAb RTE 1 detects rat keratin 19. Cytoskeletal extracts were obtained from $\left[{ }^{35} S \mid\right.$ methionine-labeled RTE cells and were subjected to two-dimensional electrophoresis. After transfer to nitrocellulose, autoradiographs were made (a), then the membrane was probed with $\mathrm{mAB}$ RTE 1 (b). The lowest molecular weight, acidic keratin is stained, which corresponds to the rat counterpart of human keratin 19.

secretory types. Basal cells appeared on GD 20 and, for the most part, were negative or only faintly expressed keratin 18 .

Keratin 19. mAb RTE 1, developed in our laboratory, stained almost all adult RTE cells (5). As shown in Figure 4, mAb RTE 1 detected the lowest molecular weight, acidic keratin in extracts of cultured RTE cells. Its position relative to other keratins and actin on the blot corresponded to that of keratin 19 as demonstrated in rat alveolar type II cells (15) and in developing rat intestinal cells (16). MAb LP2K against human keratin 19 stained the same spot on immunoblots (not shown). Therefore, RTE 1 apparently reacts with the rat homolog of human keratin 19. This is consistent with the known localization of the epitope to all simple and stratified rat epithelia examined (5).

Keratin 19 was not detectable with mAb RTE 1 in the tracheal primordium on GD 12, at which point the notochord and intestinal tube were positive (not shown). By GD 13, very faint staining was visible at the luminal border and base of a few tracheal epithelial cells (Figure 3). On GD 14 to 18, staining intensity slowly increased and was greatest in the cells overlying the membranous portion of the trachea. By GD 19, most epithelial cells of the entire trachea were strongly stained and by D 1 and D 7 the staining pattern was similar to the adult. From D 1 onward, most basal cells contained keratin 19 but a subpopulation was negative or only faintly stained with $\mathrm{mAb}$ RTE 1 .

$m A b$ RTE 3. The antigen for MAb RTE 3, which was restricted to cilia and the apical plasma membrane of ciliated cells in normal adult rat trachea (5), first appeared in a few epithelial cells overlying the membranous area on GD 18 (Figure 5). These cells corresponded to the presumed preciliated cells detected at this time on methacrylate sections.

Figure 5. mAb RTE 3 antigen expression during tracheal development. Immunoperoxidase-stained sections, methyl green counterstain. Bar $=20 \mu \mathrm{m}$. GD 18 to D 1: membranous area; D 7 to 21 : cartilaginous area.
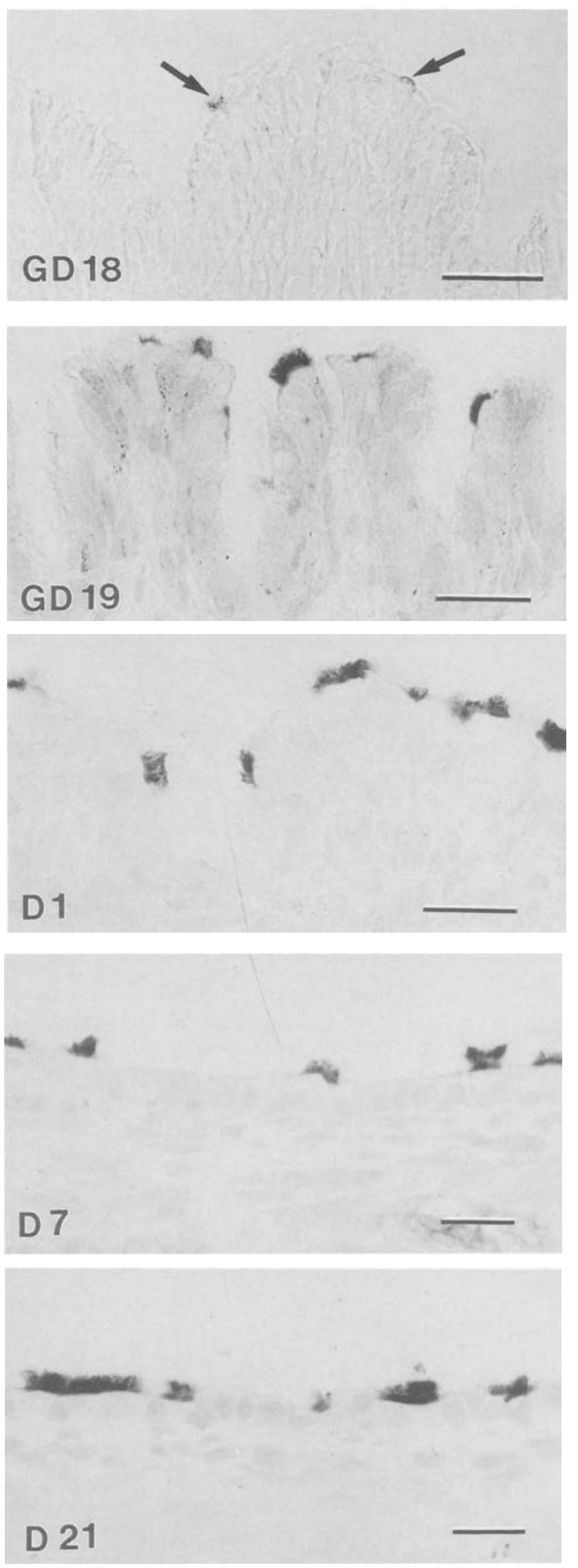

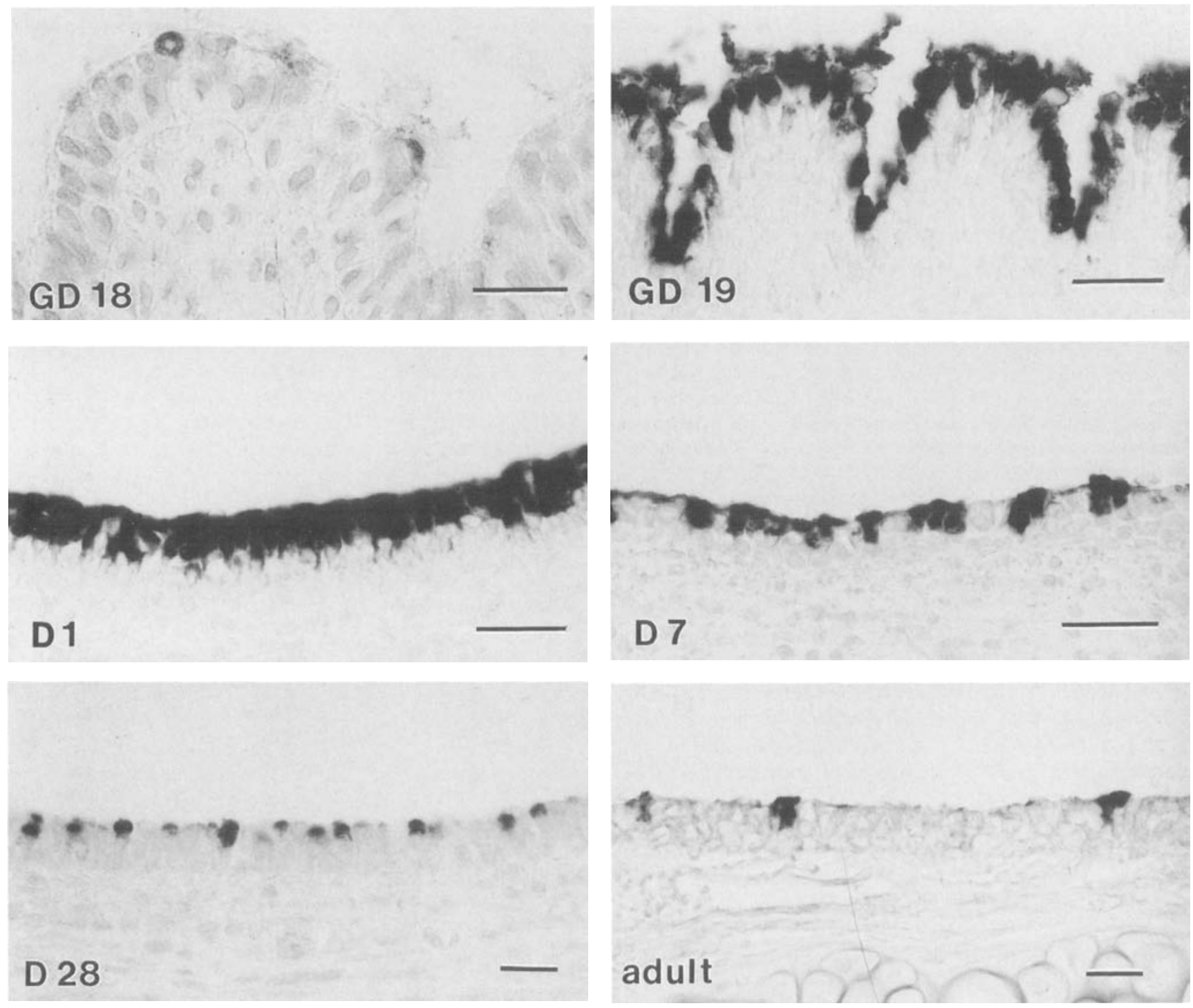

Figure 6. mAb RTE 11 antigen expression during tracheal development. Immunoperoxidase-stained sections, methyl green counterstain. Bar $=20 \mu \mathrm{m}$. GD 18 to 19 : membranous area; D 1 to adult: cartilaginous area.

The apical plasma membrane of the presumed preciliated cells did not yet have visible cilia but was stained with $\mathrm{mAb}$ RTE 3. Short (immature) or mature cilia and the apical plasma membrane of all ciliated cells were strongly stained with antibody RTE 3 on and after GD 19 (Figure 5).

mAbs RTE 9, 11, and 12. In adult rat trachea, mAbs RTE 9 and 11 stained all mucous-type secretory cells (as defined by Spicer and colleagues [17]), which were only a small fraction of the total tracheal secretory cells (5). In fetal and neonatal rat tracheas, immunoreactivity with mAbs RTE 9 and 11 was first observed in the apical cytoplasm of a few cells overlying the membranous area on GD 18 (Figure 6). By GD 19 , there was an explosive increase in staining with these mAbs; apical secretory granules were present in almost all nonciliated columnar cells and these were heavily stained. On GD 20 to D 1, the apical cytoplasms of all nonciliated columnar cells, as well as cytoplasmic granules were diffusely stained. On D 7, diffuse cytoplasmic staining disappeared, but most secretory granules continued to stain with the antibodies. Fewer secretory cells were stained by mAbs RTE 9 and 11 after D 7; however, a greater percentage of the total secretory cells were still RTE 9- and 11-positive on D 28 than in the adult. We assume that the perinatal secretory cells were not mucous cells per se but coincidentally expressed the RTE 9 and 11 antigens.

mAb RTE 12 stained almost all tracheal surface secretory cells in the adult rat, both mucous and serous types (5). In fetal and neonatal rats, immunoreactivity with mAb RTE 12 first appeared in the apical cytoplasm of scattered cells on D 1 (Figure 7). The relative frequency of mAb RTE 12reactive secretory cells increased after $D 7$, but on $D 28$ the number of secretory cells stained by this antibody was still less than in adult trachea.

$G S I-B_{4}$ lectin. GS I-B $B_{4}$ lectin is a sensitive and relatively specific marker for basal cells in adult trachea (see MATERIALS AND METHODs and reference 4). On GD 18, diffuse GS $\mathrm{I} \mathrm{B}_{4}$ lectin binding sites first appeared in the cytoplasm of columnar cells and the plasma membrane of most cells was 

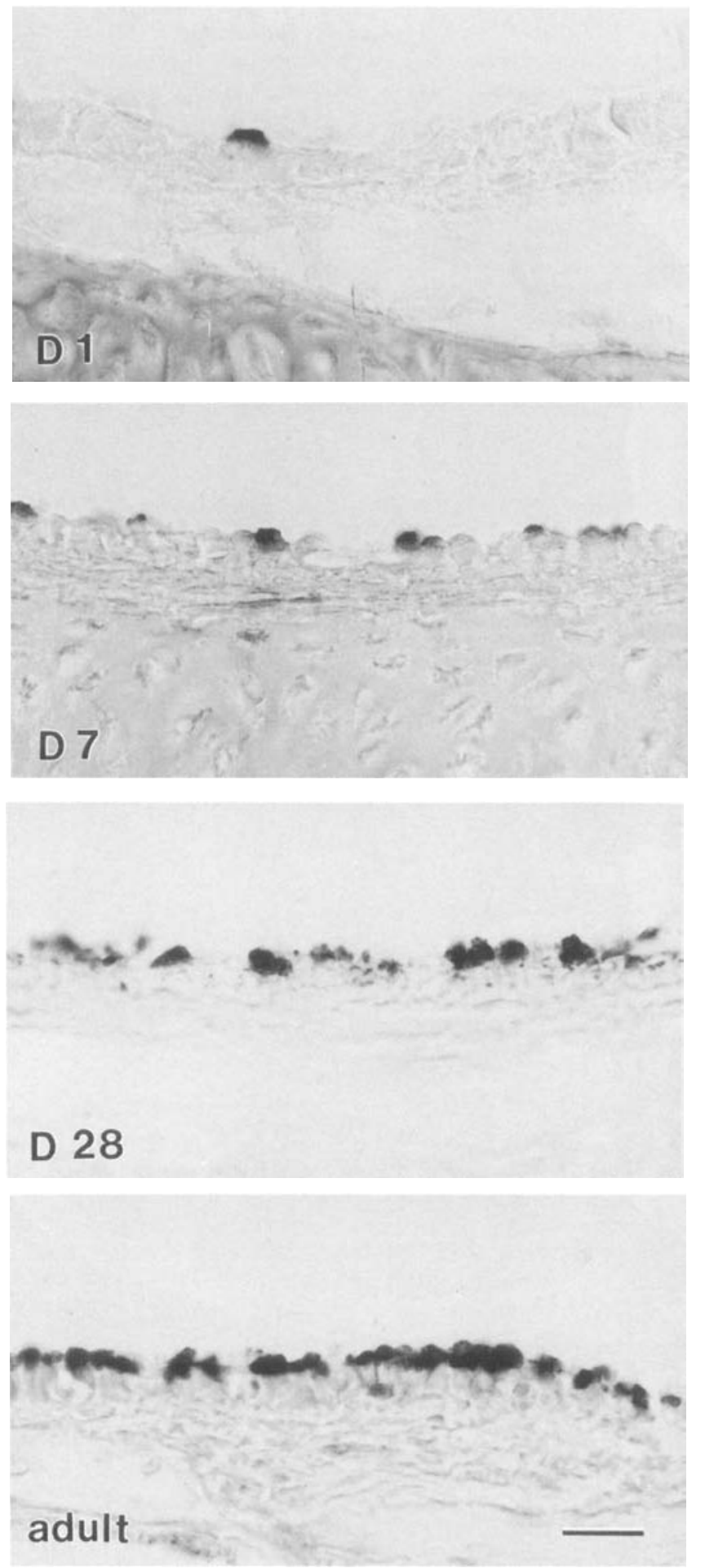

Figure 7. mAb RTE 12 antigen expression during tracheal development. Immunoperoxidase-stained sections, methyl green counterstain. Bar $=20 \mu \mathrm{m}$. All panels represent cartilaginous area.

faintly stained (Figure 8). From GD 19 to D 1, there was a large increase in lectin staining of secretory cell cytoplasms and granules, which paralleled the increase in immunostaining observed with the mAbs RTE 9 and 11 noted above. Secretory cell staining persisted until D 7, but by D 14 the relative frequency of secretory cells displaying diffuse cyto- plasmic staining decreased and secretory granules were los-

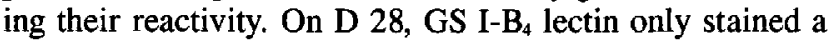
small percentage of tracheal secretory cells, similar to the adult pattern. A band of cells just above the basal lamina which were stained with GS I-B H $_{4}$ lectin became apparent on GD 20. Basal cells were consistently labeled on and after D 1. Ciliated cells were usually negative for GS $I-B_{4}$ lectin at all times.

Keratin 14. mAb LL002 reacts with human keratin 14 (10) and specifically stains basal cells in adult rat trachea (6). The nascent basal cells first seen as the epithelium became pseudostratified on GD 20 were not stained with $\mathrm{mAb}$ LL002 (Figure 8). However, by D 1 most morphologically recognizable basal cells were reactive. All basal cells were strongly positive on and after D 7. No other cell types were stained with this antibody.

mAbs RTE 2, 7, and 13. In the adult rat, mAbs RTE 2, 7 , and 13 stained most nonciliated cells (basal and secretory cells) in the upper trachea, but only reacted with a few cells in the lower trachea (5). These antibodies labeled a very prominent $69 \mathrm{kD}$ protein band on Western blots of adult RTE cells (5). Staining was observed in the cytoplasm of nonciliated cells only after D 14 (not shown).

\section{Discussion}

Using a panel of $\mathrm{mAbs}$ and a lectin, we examined the expression of phenotypic markers during fetal and neonatal tracheal development. In agreement with earlier studies $(1,2)$, the common precursor of all the major tracheal epithelial cell types during development was an undifferentiated columnar cell. Undifferentiated columnar epithelial cells in the tracheal primordium first observed on GD 12 expressed keratin 18 , which was found predominantly in columnar cells (both secretory and ciliated cells) of the adult. Keratin 19, which was present in almost all adult RTE cells, was detected, albeit faintly, on GD 13. Keratin 18 and 19 protein content increased from GD 13 onward, and they were both ubiquitous in the epithelium by GD 19. As basal cells appeared on GD 20 , they lacked or only weakly expressed keratin 18 but many contained keratin 19 . Keratins 18 and 19 were found in bronchial and lung epithelial cells as early as week 9 or 10 during the pseudoglandular stage of lung development in humans (3) and at GD 80 (48\% of term) in developing rhesus monkey airways (18); earlier times were not evaluated. In epithelial cells from developing distal rat lung, the same sequence of keratin expression we observed, namely keratin 18 before keratin 19, was seen, but at later stages (15). To our knowledge, our studies are the first to document the presence of keratin 18 in the budding endoderm as the primordial tracheal and lung epithelium first formed and the later onset of keratin 19 protein synthesis in the developing airways.

Ciliated and secretory cells, expressing specific phenotypic markers, were visible on GD 18. There was a rapid increase in secretory cells expressing the antigen for mAbs RTE 9 and 11 and a more gradual increase in ciliated cells expressing the epitope for mAb RTE 3 from GD 18 to D 1 . Basal cells were reliably detected on GD 20; they never expressed markers of highly differentiated ciliated or secretory cells. The nascent basal cells on GD 20 also did not contain keratin 14, which was first detected on D 1. Our results 
GSI-B $_{4}$
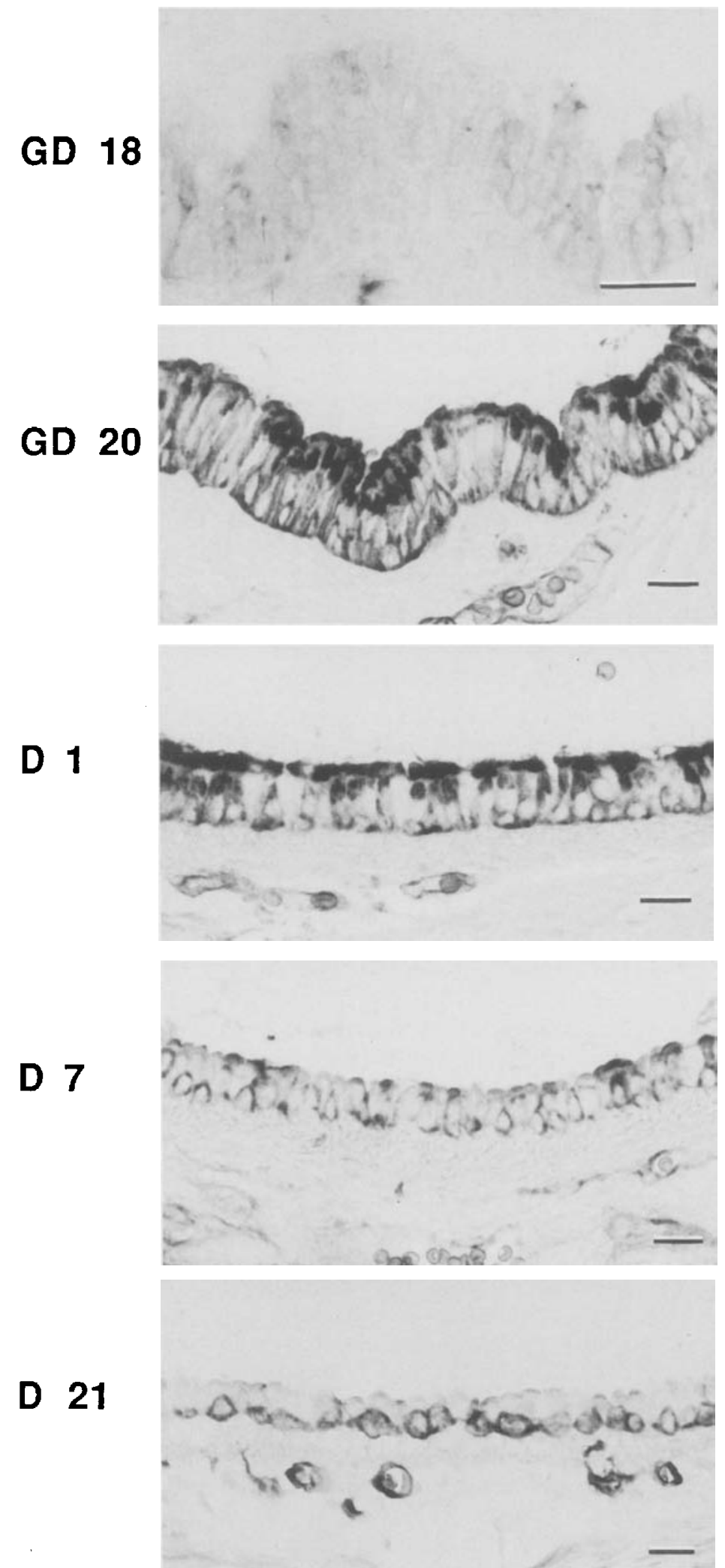

Keratin 14
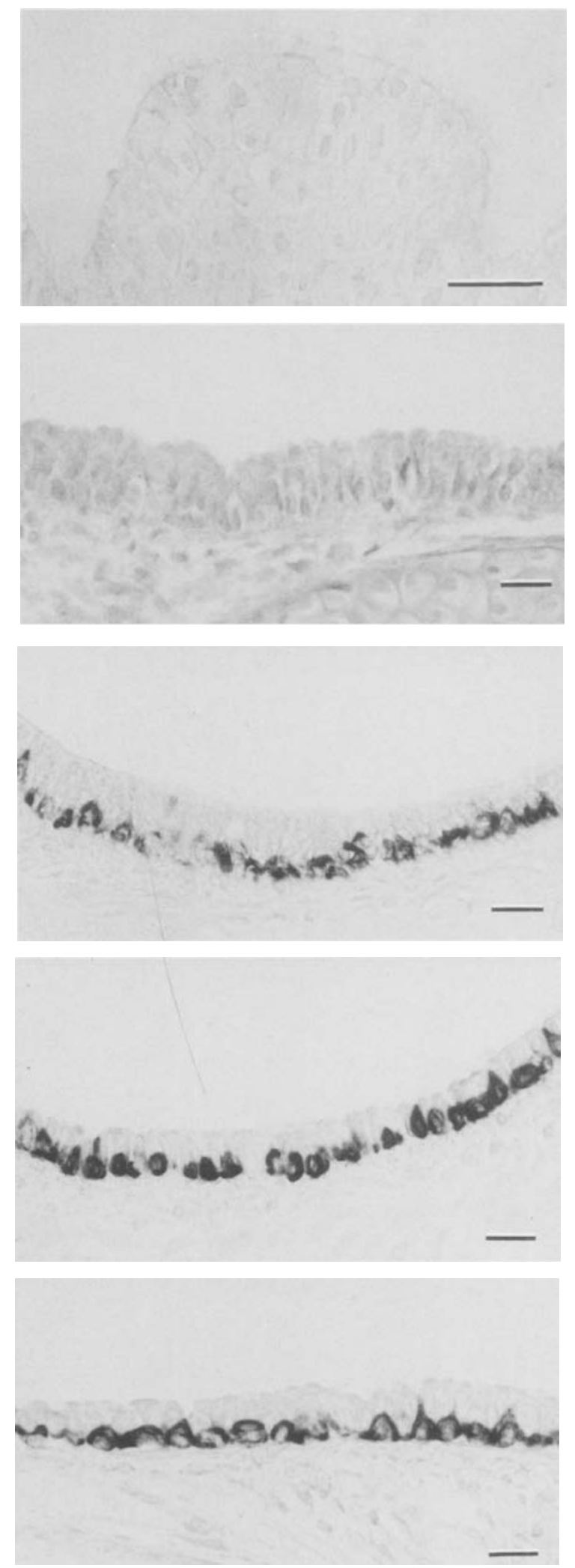

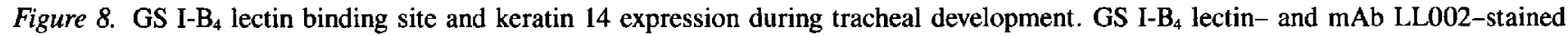

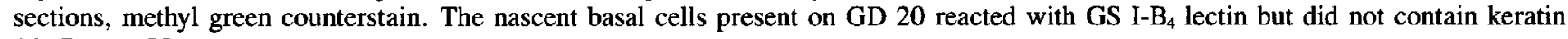
14. $\mathrm{Bar}=20 \mu \mathrm{m}$. 
clearly confirmed (3) that typical, keratin 14-containing, basal cells were not the progenitors of secretory and ciliated cells during development. It is unknown whether basal cells developed directly from undifferentiated cells, from early secretory cells, or from both.

mAbs RTE 9 and 11 and GS I-B ${ }_{4}$ lectin only react with a minority of secretory cells in the adult rat trachea and bronchus, principally with mucous cells $(4,5)$. However, from GD 19 to D 7 in developing tracheas, virtually all secretory cells were intensely stained with all three reagents. Additionally, on GD 20 and D 1 the cytoplasms as well as secretory granules contained all three markers. In contrast, mAb RTE 12 stained all tracheal surface secretory cells in the adult rat (5), but its epitope did not appear until D 1 and the number of positive cells only slowly increased to adult levels. These results demonstrate major changes in the biochemical composition of secretory cells during development and postnatal maturation. The intense staining of the cytoplasm as well as storage granules also suggests very active synthesis of secretory products during the perinatal period. Similar alterations in secretory cells were also observed during epithelial regeneration in tracheal grafts (7). These observations demonstrate the highly variable phenotype of tracheobronchial secretory cells. GS I-B ${ }_{4}$ lectin, and probably mAbs RTE 9 and 11 , detect carbohydrate moieties of mucin glycoproteins. We do not know if the changes we observed reflect variable glycosylation of the peptide backbone of the same gene product, the synthesis of novel mucin glycoprotein genes, or both. There are still large gaps in our knowledge of the specific products of tracheobronchial secretory cells, and regulation of their expression and post-translational modification.

One purpose of the present studies was to compare the sequence of marker expression between development and epithelial regeneration. At early time points during epithelial regeneration in tracheal grafts, all cells were squamoid, poorly differentiated, and contained keratin 14 and diffuse GS I- $\mathrm{B}_{4}$ lectin binding sites. At later time points, keratin 14 and diffuse GS I-B $\mathrm{B}_{4}$ lectin binding sites were gradually lost from all but basal cells as highly differentiated secretory and ciliated cell markers (mAb RTE 9 and 11 and RTE 3 antigens, respectively) were acquired (7). In grafts, nascent secretory and ciliated cells still contained keratin 14 and diffuse GS I- $\mathrm{B}_{4}$ lectin binding sites, indicating that they descended from the PD cells (7). In developing tracheas, the epitopes detected by mAbs RTE 9 and 11 and RTE 3 appeared before keratin 14 . Regeneration has been generally thought of as recapitulating the steps taken during development. We observed similar patterns of increasing keratin 18, and secretory and ciliated cell antigen expression in both development and regeneration. Interestingly, keratin 14 expression was strikingly different between these processes, revealing a fundamental difference in pathways of cellular biochemical differentiation. Perhaps one sequence is fol- lowed for the initial developmental acquisition of the major tracheal epithelial cell phenotypes, but, once established, new and different pathways become operative.

Acknowledgments: The writers acknowledge the expert technical assistance of the NIEHS histology laboratory and thank the NIEHS in-house breeding program for providing precisely timed pregnant rats. We thank Dr. E. B. Lane (Dundee, UK) for kindly providing antibodies LL002 and LP2K.

\section{References}

1. McDowell, E. M., C. Newkirk, and B. Coleman. 1985. Development of hamster tracheal epithelium. II. Cell proliferation in the fetus. Anat. Rec. 213:448-456.

2. Plopper, C. G., J. Alley, and A. J. Weir. 1986. Differentiation of tracheal epithelium during fetal lung maturation in the rhesus monkey Macaca mulatta. Am. J. Anat. 175:59-71.

3. Broers, J. L. V., L. de Leij, M. Klein Rot, A. Terttaar, E. B. Lane I. M. Leigh, S. S. Wagenaar, G. P. Vooijs, and F. C. S. Ramaekers 1989. Expression of intermediate filament proteins in fetal and adult human lung tissues. Differentiation 40:119-128.

4. Shimizu, T., P. Nettesheim, J. G. Mahler, and S. H. Randell. 1990. Celltype specific lectin staining of the tracheobronchial epithelium of the rat: quantitative studies with Griffonia simplicifolia I isolectin B4. J. Histochem. Cytochem. 39:7-14.

5. Shimizu, T., P. Nettesheim, E. M. Eddy, and S. H. Randell. 1992. Monoclonal antibody (Mab) markers for subpopulations of rat tracheal epithelial (RTE) cells. Exp. Lung Res. 18:323-342.

6. Randell, S. H., C. E. Comment, F. C. S. Ramaekers, and P. Nettesheim. 1991. Properties of rat tracheal epithelial cells separated based on expression of cell surface $\alpha$-galactosyl end groups. Am. J. Respir. Cell Mol. Biol. 4:544-554.

7. Shimizu, T., P. Nettesheim, F. C. S. Ramaekers, and S. H. Randell. 1992. Expression of "cell-type-specific" markers during rat tracheal epithelial regeneration. Am. J. Respir. Cell Mol. Biol. 7:30-41.

8. Moll, R., W. W. Franke, D. L. Schiller, B. Geiger, and R, Krepler. 1982. The catalog of human cytokeratins: patterns of expression in normal epithelia, tumors and cultured cells. Cell 31:11-24.

9. Broers, J. L. V., D. N. Carney, M. Klein Rot, G. Schaart, E. B. Lane, G. P. Vooijs, and F. C. S. Ramaekers. 1986. Intermediate filament proteins in classic and variant types of small cell lung carcinoma cell lines: a biochemical and immunochemical analysis using a panel of monoclonal and polyclonal antibodies. J. Cell Sci. 83:37-60.

10. Purkis, P. E., J. B. Steel, I. C. McKenzie, W. B. J. Nathrath, I. M. Leigh, and E. B. Lane. 1990. Antibody markers of basal cells in complex epithe lia. J. Cell Sci. 97:39-50.

11. Kaartinen, L., P. Nettesheim, K. B. Adler, and S. H. Randell. 1993. Rat tracheal epithelial (RTE) cell differentiation in vitro. In Vitro Cell Mol. Biol. (In press)

12. Achtstaetter, T., M. Hatzfeld, R. A. Quinlan, D. C. Parmelee, and W. W. Franke. 1986. Separation of cytokeratin polypeptides by gel electrophoretic and chromatographic techniques and their identification by immunoblotting. In Methods in Enzymology. Vol. 134. R. B. Vallee, editor. Academic Press, Orlando, FL. 355-371.

13. Dunbar, B. S. 1987. Two-dimensional electrophoresis and immunological techniques. Plenum Press, New York. 103-115, 217-220.

14. Lane, E. B., J. Bartek, P. E. Purkis, and I. M. Leigh. 1985. Keratin antigens in differentiating skin. Ann. NY Acad. Sci. 455:241-258.

15. Paine, R., A. Ben-Ze'ev, S. R. Farmer, and J. S. Brody. 1988. The pattern of cytokeratin synthesis is a marker of type 2 cell differentiation in adult and maturing fetal lung alveolar cells. Dev. Biol. 129:505-515.

16. Quaroni, A., D. Calnek, E. Quaroni, and J. S. Chandler. 1991. Keratin expression in rat intestinal crypt and villus cells - analysis with a panel of monoclonal antibodies. J. Biol. Chem. 266:11923-11931.

17. Spicer, S. S., I. Mochizuki, M. E. Setser, and J. R. Martinez. 1980. Complex carbohydrates of rat tracheobronchial surface epithelium visualized ultrastructurally. Am. $J$. Anat. 158:93-109.

18. Huang, T. H., J. A. St. George, C. G. Plopper, and R. Wu, 1989. Keratin protein expression during the development of conducting airway epithelium in nonhuman primates. Differentiation 41:78-86. 